\title{
High Prevalence of Epilepsy in Onchocerciasis Endemic Com- munities of Ngie: Lack of Association with Taeniasis but Strong Indicators of Hereditary Factors and Characteristics of Oncho- cerciasis Associated Epilepsy Observed
}

\author{
Raphael Awah Abong ${ }^{1,2^{*}}$, Fongoh Ayong Hassan ${ }^{1}$, Tatsinkou Betrand Fossi ${ }^{1}$, Peter Ndefon ${ }^{3}$, Theresa \\ Nkuo-Akenji ${ }^{1}$, Samuel Wanji, ${ }^{1,2}$ and Anong Damian Nota ${ }^{1}$
}

${ }^{1}$ Department of Microbiology \& Parasitology, Faculty of Science, University of Buea, Cameroon

${ }^{2}$ Research Foundation in Tropical Diseases and Environment (REFOTDE), PO Box 474, Buea, Cameroon

${ }^{3}$ Department of Public Health \& Hygiene, Faculty of Health Science, University of Buea, Cameroon

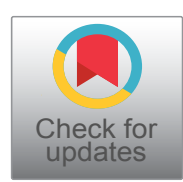

*Corresponding author: Raphael Awah Abong, Research Foundation in Tropical Diseases and Environment (REFOTDE), PO Box 474, Buea, Cameroon, Tel: +237674609504

\begin{abstract}
Background: Epilepsy, a neurological condition which affects individuals of all ages has been widely reported to be caused by parasitic, fungal, bacterial or viral infectious diseases of the Central Nervous System (CNS). Taenia solium infection otherwise known as neurocysticercosis in taeniasis endemic zones, Onchocerca volvulus infection in onchocerciasis meso/hyperendemic areas in Africa and other infectious agents have been highly incriminated in different parts of the world. Epilepsy remains an important but neglected public health problem in many countries. Ngie sub division is endemic for onchocerciasis and the people are highly involved in pig farming and pork consumption. This study aimed to determine the prevalence of epilepsy in Ngie, investigate its association with Taenia solium infection as well as other risk factors.

Methods: A stratified random sampling in which six villages were selected from three clusters was used for the study. Nine hundred and fourteen (914) structured questionnaires were administered in a community-based door-to-door survey to determine the prevalence of epilepsy and its associated risk factors. Stool, urine and blood samples from 298 individuals were studied by microscopy in a case-control community based laboratory investigation to establish the relationship between epilepsy and $T$. solium as well as other intestinal, blood and urinary parasites. Infection with $O$. volvulus was not investigated.
\end{abstract}

Results: The prevalence of epilepsy in this population was $9 \%$ (82/914). Infection by Taenia species was $1.5 \%(2 / 135)$ in epilepsy patients and $0.6 \%(1 / 163)$ in non-epilepsy participants $(P>0.5)$. Yeast cells were significantly higher in the stool of epilepsy patients $(14.1 \%)$ than the controls $(5.5 \%)(P=0.016)$. Among the risk factors investigated in this study, a positive family history $(93.9 \%$ for epileptics and $42.5 \%$ for non-epileptics) and fungal infection could be incriminated. The peak age of epilepsy onset in patients was 12 years $(19.5 \%, 16 / 82)$ and over $95 \%$ of the patients did not exceed 30 years of age. Characteristics of Onchocerciasis Associated Epilepsy (OAE) where observed.

Conclusion: The high prevalence of epilepsy in Ngie sub division in the Momo valley is not associated with $T$. solium infection. Family history, fungal infection and $O$. volvulus infection could be responsible.

\section{Keywords}

High-prevalence, Epilepsy, Taeniasis, Family-history, Onchocerciasis, Ngie

\section{Introduction}

Epilepsy, defined as "recurrent unprovoked seizures that occur due to abnormal electrical activity in the brain" [1], is one of the most common neurological conditions worldwide. Anything that can cause struc-

Citation: Abong RA, Hassan FA, Fossi TB, Ndefon P, Nkuo-Akenji T, et al. (2018) High Prevalence of Epilepsy in Onchocerciasis Endemic Communities of Ngie: Lack of Association with Taeniasis but Strong Indicators of Hereditary Factors and Characteristics of Onchocerciasis Associated Epilepsy Observed. Int J Trop Dis 1:010.

Accepted: November 10, 2018; Published: November 12, 2018

Copyright: (c) 2018 Abong RA, et al. This is an open-access article distributed under the terms of the Creative Commons Attribution License, which permits unrestricted use, distribution, and reproduction in any medium, provided the original author and source are credited. 
tural or functional damage of brain physiology may lead to seizures, and various conditions may express themselves solely by recurrent seizures and thus be labelled "epilepsy" [2]. The International League Against Epilepsy (ILAE) and International Bureau for Epilepsy (IBE) in 2005 as well as the World Health Organization $[1,3]$, reported that about 50 million people worldwide have epilepsy with almost $80 \%$ of these people living in low- and middle-income countries [3]. It is a major health problem in Central and West Africa [4,5] with prevalence estimates (based on door-to-door surveys) ranging between 22.1 per 1,000 in West Africa to 64.8 per 1,000 in Central Africa. Epilepsy is related to serious physical and psychological consequences, including premature death, traumatic injury and mental health disorders. Moreover, a substantial treatment gap is evident in developing countries, because human and financial resources for diagnosis and treatment are limited and misconceptions and stigma surround the disorder [4].

Various parasitic infections are thought to be involved in causing epilepsy, especially in tropical countries. These include malaria, neurocysticercosis and onchocerciasis. Neurocysticercosis (NCC) is a parasitic infection of the human central nervous system, which involves infection of the brain parenchyma with the larval form of the Taenia solium tapeworm and authors have reported that it may be associated with epilepsy [6-10]. On the other hand, a review from eight studies in West (Benin and Nigeria), Central (Cameroon and Central African Republic) and East (Uganda, Tanzania and Burundi) Africa [11] also provided evidence of an association between epilepsy and onchocerciasis (Onchocerciasis Associated Epilepsy - OAE). Recent epidemiological and clinical findings characterized OAE as a spectrum of epilepsy with onset of seizures between the ages of 3 and 18 years [12], whereas the onset of epilepsy due to NCC is primarily after the age of 25 years [13]. In Bilomo, a village found in the Centre Region of Cameroon, hereditary, perinatal conditions, CNS infections and traumatic head injury were reported as risk factors [13] while a village hyper-endemic for onchocerciasis in the Sanaga River Valley in the Littoral Region report only a positive family history as risk factor [14].

Epilepsy has become an apparent epidemic in Momo Division of the North West Region of Cameroon. In some cases, the whole family (parents and children) is affected. Pig breeding has been a traditional occupation of some communities and pork constitutes part of their food. Traditionally, pigs are important for funeral ceremonies, marriages and every kind of ritual in Momo Division. Because of high consumption of pork, and poor hygienic conditions as pigs were allowed to move freely in the communities, it was suspected that epilepsy in Ngie could be due to neurocysticercosis caused by Taenia solium. Even though a study by Wanji and colleagues declared this area hyper-endemic for onchocerciasis [15] we did not investigate onchocerciasis infection due to lack of means. This study was designed to determine the prevalence and possible causes of epilepsy in Ngie with an emphasis on the role of Taenia species.

\section{Materials and Methods}

This study was carried out in six (6) of nineteen (19) randomly selected villages of Ngie Sub Division, a rural community in Momo Valley area, North West Region of Cameroon with a population of about 18,000 inhabitants [16]. Ngie is located between latitude $50^{\circ} 43^{\prime}$ and $60^{\circ} 10^{\prime} \mathrm{N}$ and longitude $90^{\circ} 43^{\prime}$ and $90^{\circ} 55^{\prime} \mathrm{E}$ [17]. The topography is characterized by hills and valleys with some fast flowing rivers which are potential breeding sites for 0 . volvulus vectors (Simulium species). A study by Wanji, et al. [15] revealed that the Momo valley where Ngie is situated, was hyper-endemic for onchocerciasis with over $20 \%$ palpable nodule prevalence and above $60 \%$ microfilaridermia in all ten communities where screening was performed before the introduction of Ivermectin Mass Drug Administration (IVM-MDA) by the African Program for Onchocerciasis Control (APOC). Farming and pig-rearing are the main economic activities in these villages. Palm oil, Kolanuts, coffee and sales of pigs are the main sources of income. Pork is highly consumed in this community.

The Stratified Cluster Sampling (SCS) approach described by [18] was adopted for the study. Ngie Subdivision was subdivided into three clusters (Upper, Central and Lower Ngie) based on location. From each cluster, two villages were randomly selected; Tinechung and Tinekoh from upper Ngie, Ankek and Ajei from central Ngie and Teze and Bonambufei from lower Ngie. In each village, the surveyors systematically moved from Door-to-door and interviewed participants after every one household. The participants of the study were persons of both sexes and greater than or equal to 10 years of age who had lived in Ngie for at least 5 years. Nine hundred and fourteen structured questionnaires were administered to collect sociodemographic data of participants as well as data on potential risk factors for epilepsy, such as a positive family history (first degree relatives), traumatic head injury, meningitis/encephalitis, frequent consumption of pork, type of fly bite most suffered, hygienic practices, malaria history, consumption of marijuana or any other drug to stimulate body and excessive alcohol intake. Epileptics were defined as individuals who had suffered two or more unprovoked seizures within the past 12 months. Prevalence of epilepsy was calculated by dividing the number of epileptics by the total number of inhabitants interviewed in the selected villages. The prevalence of epilepsy was compared between various age groups based on intervals of 10 years.

Additionally, a case-control study was performed to assess risk factors for epilepsy. The sample size was purposive, based on the number of patients and controls in the community who voluntarily accepted to be part of 
the study and came to the different health centers where they donated samples for examination. All participants of the case control study came from the selected villages and included those whose households were not interviewed. Each patient who came to the hospital was examined alongside one or more other members of the same household as controls. Two hundred and ninety eight (298) individuals ( 135 cases and 163 controls) divided into 5 age-groups donated stool, urine and blood specimens for microscopic examination, to investigate the presence of Taenia species and other parasitic (Plasmodium species, intestinal helminths, urino-genital parasite and fungi (yeast cells)) infections excluding $O$. volvulus.

Stool, blood, and urine specimens were collected from study participants using standard procedures [19]. Stool in $10 \%$ formalin and urine specimens were taken directly to the laboratory for examination. Normal saline and iodine preparation followed by the formol-ether concentration technique were used for the diagnosis of intestinal parasites in the stool samples [19]. Wet mount preparations were observed at X10 and X40 objectives. Taeniasis was determined by the presence of egg or segment(s) of tapeworm in stool. Blood parasites (Plasmodium spp. in particular) were investigated using capillary blood collected from study participants, stained and observed according to standard procedures for malaria parasites [19].

Microsoft Excel version 2007 was used to store the data and plot graphs. Data was then transferred to SPSS version 17.0 statistical software package for analysis. The Chi square test was used to test whether significant differences in risk factors between epileptics and non-epileptics were present. Statistical significance was set at $P<0.05$ at $95 \%$ confidence interval (CI).

\section{Ethical Considerations}

The study was approved by the Regional Delegation of Public Health. Informed consent forms were signed by the study participants before enrollment in the study. Children below 18 years of age were registered for the study after an oral parental/guardian informed consent. For epileptic patients who could not express themselves properly due to the advanced state of the seizures, information was given either by their parents or any elderly family member living in the same home with the patient. All participants' information collected during the course of the research was kept on a password protected database that was strictly confidential. In addition to the individual consent, the village chiefs of the six villages in Ngie gave their authorisation for the study to be carried out in their communities.

\section{Results}

\section{Prevalence of epilepsy in Ngie}

Of the 914 persons who answered the questionnaires, 82 were suffering from epilepsy, corresponding to an overall epilepsy prevalence of $9.0 \%$ (82/914) in the selected villages of Ngie. However, there was a significant difference in the prevalence of epilepsy in the different clusters $\left(x^{2}=53.503, P<0.001\right)$. From the 304,310 and 300 individuals interviewed in the Tinechung-Tinekoh (T-T), Andek-Ajei (A-A) and Teze-Bonambufei (T-B) clusters, $2.0 \%(6 / 304), 6.5 \%(20 / 310)$ and $18.4 \%$ (56/300) were epileptics respectively.

\section{Sociodemographic characteristics of participants}

The sociodemographic information of the respondents to the questionnaire is shown in Table 1.

Table 1: Sociodemographic data on participants.

\begin{tabular}{|c|c|c|c|c|c|}
\hline \multicolumn{2}{|l|}{ Parameters } & Epileptics (82) & Non Epileptics (832) & $\left(X^{2}\right)$ & P-value \\
\hline \multicolumn{2}{|c|}{ Age range (years) } & $10-50$ Mean $=17.73 ;$ Median $=17$ & $10-80$ Mean $=29.68 ;$ Median $=26$ & & \\
\hline \multirow[t]{3}{*}{ Place of birth } & Ngie & $85.4 \%(70 / 82)$ & $77.4 \%(644 / 832)$ & & \\
\hline & Within Momo & $6.1 \%(5 / 82)$ & $8.1 \%(67 / 832)$ & & \\
\hline & Out of Momo & $8.5 \%(7 / 82)$ & $14.4 \%(120 / 832)$ & & \\
\hline \multirow[t]{2}{*}{ Gender } & Males & $57.3 \%(47 / 82)$ & $48.3 \%(402 / 832)$ & \multirow[t]{2}{*}{2.419} & \multirow[t]{2}{*}{0.133} \\
\hline & Females & $42.7 \%(35 / 82)$ & $51.7 \%(430 / 832)$ & & \\
\hline \multicolumn{2}{|c|}{ Pit Latrine present at home } & $87.8 \%(72 / 82)$ & $84.9 \%(706 / 832)$ & 1.529 & 0.466 \\
\hline \multirow[t]{3}{*}{ Status } & Married & $11.0 \%(9 / 82)$ & $49.9 \%(415 / 832)$ & & \\
\hline & Living with parents & $87.9 \%(72 / 82)$ & $38.7 \%(322 / 832)$ & & \\
\hline & Living alone & $1.2 \%(1 / 82)$ & $11.1 \%(92 / 832)$ & & \\
\hline \multirow[t]{4}{*}{ Literacy level } & None & $0 \%(0 / 82)$ & $6.3 \%(52 / 832)$ & & \\
\hline & Primary & $74.4 \%(61 / 82)$ & $42.2 \%(351 / 832)$ & & \\
\hline & Secondary & $25.6 \%(21 / 82)$ & $49.0 \%(408 / 832)$ & & \\
\hline & Above secondary & $0 \%(0 / 82)$ & $2.5 \%(21 / 832)$ & & \\
\hline \multirow[t]{8}{*}{ Profession } & Farmer & $42.7 \%(35 / 82)$ & $43.3 \%(360 / 832)$ & & \\
\hline & Salaried employee & $1.2 \%(1 / 82)$ & $7.7 \%(64 / 832)$ & & \\
\hline & Students & $17.1 \%(14 / 82)$ & $33.1 \%(275 / 832)$ & & \\
\hline & Business & $0 \%$ & $6.0 \%(50 / 832)$ & & \\
\hline & Herdsmen & $0 \%$ & $0.5 \%(4 / 832)$ & & \\
\hline & House works & $35.4 \%(29 / 82)$ & $5.8 \%(48 / 832)$ & & \\
\hline & Labourers & $2.4 \%(2 / 82)$ & $3.2 \%(27 / 832)$ & & \\
\hline & Craftsmen & $1.2 \%(1 / 82)$ & $0.2 \%(2 / 832)$ & & \\
\hline
\end{tabular}




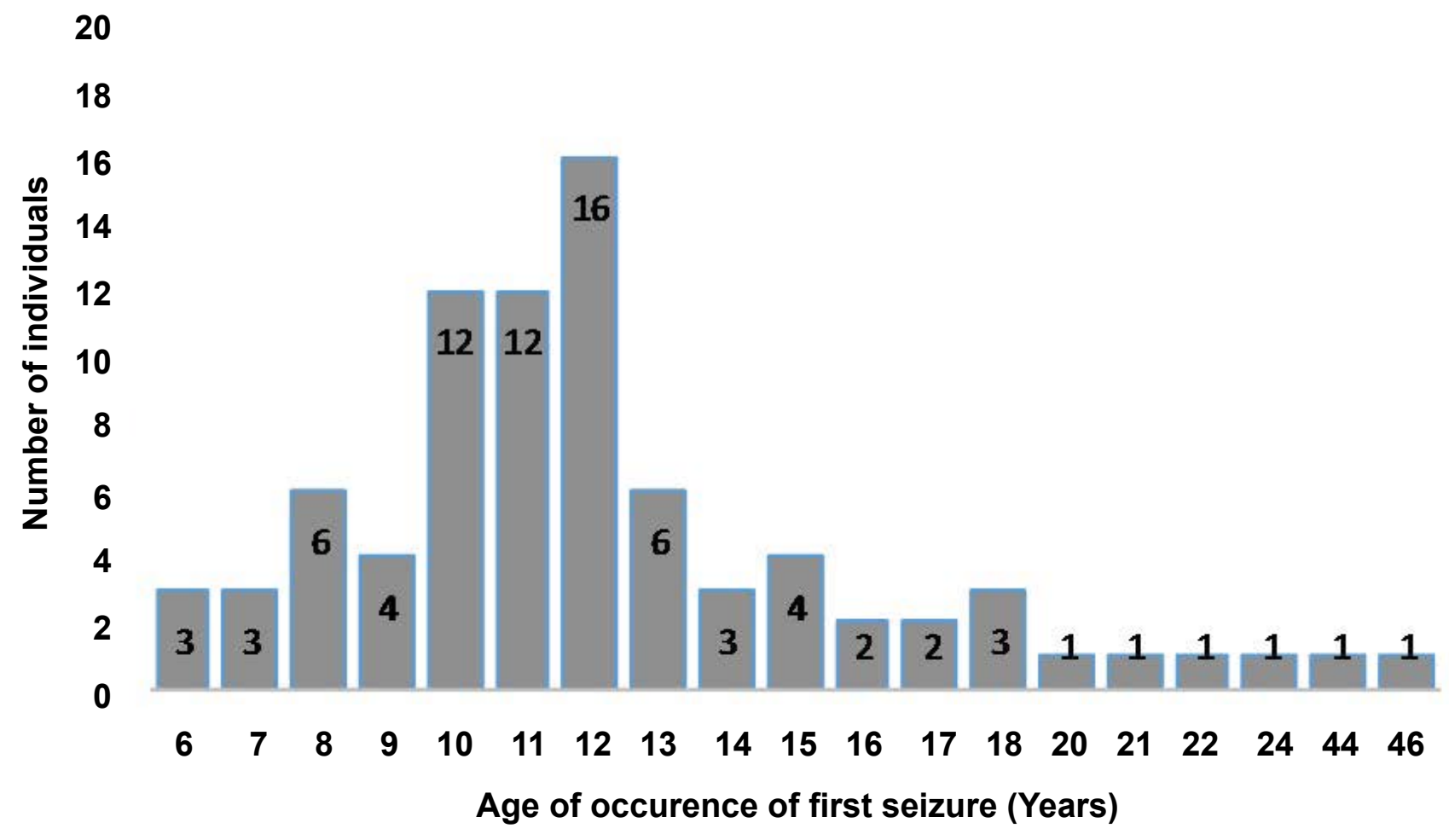

Figure 1: Age of occurrence of first epileptic seizure among epileptic patients in Ngie.

Table 2: Age-group distribution of participants in the case-control community based study.

\begin{tabular}{|c|c|c|c|c|c|}
\hline Age-group (years) & Epilepsy patients & Non-epilepsy persons & Total & $\mathbf{X}^{2}$ & P-value \\
\hline $10-20$ & $79 / 135(58.5 \%)$ & $45 / 163(27.6 \%)$ & $124 / 298(41.6 \%)$ & \multirow[t]{6}{*}{76.402} & \multirow[t]{6}{*}{$<0.001$} \\
\hline $21-30$ & $53 / 135(39.3 \%)$ & $42 / 163(25.8 \%)$ & 95/298 (31.9\%) & & \\
\hline $31-40$ & $0 / 135(0 \%)$ & $31 / 163(19.0 \%)$ & $31 / 298(10.4 \%)$ & & \\
\hline $41-50$ & $3 / 135(1.5 \%)$ & $27 / 163(16.6 \%)$ & $30 / 298(10.1 \%)$ & & \\
\hline$>50$ & $0 / 135(0.7 \%)$ & $18 / 163(11.0 \%)$ & $18 / 298(6.0 \%)$ & & \\
\hline Total & $135 / 298(45.3 \%)$ & $163 / 298(54.7 \%)$ & $298 / 298(100 \%)$ & & \\
\hline
\end{tabular}

Age of occurrence of first epileptic seizure among epileptic patients

From our questionnaire respondents, we determined that, the age at which patients in this study area experienced the first seizure(s) ranged between 6 and 46 years. The mean age of all 82 epileptic patients was 12.73 years with 12 years as modal age and a standard deviation of 6.230. Most of these patients 77 (93.9\%), experienced the first seizure before the age of 20 years with $16(19.5 \%)$ of them experiencing epilepsy at the age of 12 years (Figure 1). Only $5(6.1 \%)$ of the patients started experiencing the seizures after 20 years of age.

\section{Age-group distribution among the case-control participants}

The age of epilepsy patients who came for the case-control study ranged from 10 to 50 -years-old ( $\mathrm{Ta}$ ble 2) while the age of controls ranged between 10 and 67-years-old. Most of the cases were within the 10-20 years age group and there was a statistically significant difference in their distribution in the different age groupings $\left(x^{2}=76.402, P<0.001\right)$. Out of the 135 epilepsy patients who came for the case control study, only 3 of them were within the age group of $40-50$ years and none was above 50-years-old. One hundred and thirty two (97.8\%) of the patients/cases who donated samples for the case control study were within $10-30$ years old. The age-group of the 163 control participants differed from that cases (Table 2), however, they have the same living conditions and environmental exposure to pathogens.

\section{Distribution of Taenia species in the stool of par- ticipants}

Out of the 298 stool samples investigated, $1.0 \%$ (3/298) had Taenia ova/eggs (2/135 of epileptics and $1 / 163$ of non-epileptics). There was no significant difference in the presence of Taenia ova in epileptics when compared with non-epileptics $\left(x^{2}=0.558, p=0.592\right)$.

\section{Distribution of intestinal parasites other than Tae- nia species in stool of participants}

There was no significant difference in the distribution of other intestinal parasites found in epileptic patients compared with non-epileptic patients (Table 3).

\section{Presence of parasites in urine of participants}

Out of the 298 urine samples analyzed, no parasites 
Table 3: Distribution of intestinal parasites other than Taenia species in stool of participants.

\begin{tabular}{|c|c|c|c|c|c|}
\hline Parasite eggs & Epileptics & Non Epileptics & Total & $\mathbf{x}^{2}$ & P-value \\
\hline Ascaris lumbricoides & $23(17.0 \%)$ & $21(12.9 \%)$ & $44(14.77 \%)$ & 1.012 & 0.329 \\
\hline Hookworm & $9(6.7 \%)$ & $7(4.3 \%)$ & $16(5.37 \%)$ & 0.818 & 0.442 \\
\hline Entamoeba coli & $7(5.2 \%)$ & $9(5.5 \%)$ & $16(5.37 \%)$ & 0.016 & 1.000 \\
\hline Entamoeba histolytica & $8(5.9 \%)$ & $13(8.0 \%)$ & $21(7.05 \%)$ & 0.474 & 0.650 \\
\hline Trichuris trichiura & $2(1.5 \%)$ & $1(0.6 \%)$ & $3(1.01 \%)$ & 0.558 & 0.592 \\
\hline Hymenolepis diminuta & $3(2.2 \%)$ & $0(0.0 \%)$ & $3(1.01 \%)$ & 3.659 & 0.092 \\
\hline Schistosoma species & $2(1.5 \%)$ & $0(0.0 \%)$ & $2(0.67 \%)$ & 2.431 & 0.204 \\
\hline Trichomonas species & $1(0.7 \%)$ & $0(0.0 \%)$ & $1(0.34 \%)$ & 1.211 & 0.453 \\
\hline Entamoeba intestinalis & $0(0.0 \%)$ & $1(0.6 \%)$ & $1(0.34 \%)$ & 0.831 & 1.000 \\
\hline Yeast cells & $19(14.1 \%)$ & $9(5.5 \%)$ & $28(9.4 \%)$ & 6.345 & 0.016 \\
\hline
\end{tabular}

were seen in the urine of both epileptics and non-epileptics.

\section{Presence of blood parasites in participants}

Of the 298 blood specimens from subjects (cases and controls) examined, the malaria prevalence was higher $\left(x^{2}=10.053\right.$, and $\left.P=0.002\right)$ among non-epileptics (48.5\% i.e. $79 / 163$ ) compared to that among epileptics (30.4\% i.e. 41/135). This shows that malaria is not associated with epilepsy in this area. The total prevalence of malaria parasites in the study population was $40.3 \%$ (120/298). No other blood borne parasites were found in patients by microscopy.

\section{Associated risk factors of epilepsy}

Our study showed that $95.1 \%$ (78/82) of patients had a positive family history of epilepsy as opposed to $42.5 \%$ (354/832) of non-patients. Of all the patients, $2.4 \%(2 / 82)$ suffered from the disease after serious head injury, $1.2 \%(1 / 82)$ had onset after suffering from severe meningitis (central nervous system infection) and $1.2 \%$ $(1 / 82)$ suffered onset because of excessive alcohol use (intoxication).

\section{Discussion}

The primary purpose of this study was to determine the prevalence of epilepsy in Ngie, determine any association with taeniasis and investigate other risk factors that may predispose individuals to epilepsy in the area. The observed prevalence of $9 \%$ is very high when compared to the prevalence reported in tropical countries (1.0-1.5\%) [20] and in Cameroon (1-4\%, [9] and $4.9 \%$ [13]). However, our prevalence is very close to the $10.5 \%$ reported in a small village located in a geographically isolated area, hyper-endemic for onchocerciasis in the Littoral Region of Cameroon [14].

The significant difference in the prevalence of epilepsy in the different study clusters of Ngie $\left(x^{2}=53.503, P=\right.$ 0.000 ) is similar to patterns that are often seen between communities with different degrees of onchocerciasis endemicity within an endemic focus where people suffer from OAE as reviewed [11]. It is important to note that the cluster of Teze-Bonambufei, which showed the highest prevalence, has more fast flowing rivers very close to human settlements compared to the other two clusters.
We expected to find a significant number of individuals in the population with Taenia ova or segments in their stool so as to establish a possible link with epilepsy. The very low prevalence of Taenia species in the population and lack of association with the presence of epilepsy in the community is an indication that the disease in Ngie may be due to factors other than taeniasis. This very low prevalence of taeniasis is very similar to the $0 \%$ reported in an epilepsy endemic area in Bas-Ue' le' Province, Democratic Republic of the Congo [21]. Infection with malaria parasite was not associated with epilepsy.

A positive family history could be a major risk factor of epilepsy in this area, as it was present in $95.1 \%(78 / 82)$ of the epileptic patients compared to $42.5 \%$ (354/832) of the non-epileptics. From other studies in Cameroon, a positive family history was the main risk factor for epilepsy in the Sanaga valley [14] and was also highlighted in Bilomo village [13]. A man from Lower Edem in Teze village and two of his children from separate wives together with some of his brother's off springs were epileptic. There were several households were either 5 of 8,3 of 5 and a nephew, and 3 of 6 children of the same parents suffered from epilepsy. This shows that genetic factors may likely be associated with epilepsy in this area and not Taenia as reported in other studies $[9,10]$. From an environmental perspective, the high number of epilepsy cases clustered in some families and villages is a characteristic of OAE as all family members and villagers are exposed to $O$. volvulus infected black flies close to rapid-flowing rivers [12]. However, we did not investigate onchocerciasis as a risk factor in this study.

The American Epilepsy Society (2010) reported that anyone can develop epilepsy despite the age, race and social class. The onset of epilepsy in Ngie in $93.9 \%$ $(77 / 82)$ of epileptics was between the ages of 6 to 20 years with $19.5 \%(16 / 82)$ of them experiencing first seizures at the age of 12 years. Similar findings have been reported in areas endemic for onchocerciasis, and the age at onset of seizures is mentioned as a characteristic of OAE [12].

For the patients that reported for the case control study, we observed that over $95 \%$ of them were within the age groups of $10-20$ years (58\%) and $21-30$ years 
(39\%) while less than $5 \%$ were above 30 years of age. This is an indication that, most of the epileptic patients in this community may die before they can exceed 30 -years-old. This finding corroborate a previous study [22] who reported that epileptic patients in onchocerciasis endemic regions experience high mortality rates. Between the peak age of seizure onset (12-years-old) and max 30 years of age for over $95 \%$ of patients, will gives us approximately 18 years that most epilepsy patients live after experiencing the first seizure.

The significantly higher prevalence of yeast cells in the stool of epilepsy patients $(P=0.016)$ is a signal that, epilepsy in some of the patients could be associated with fungal infections that affect the CNS [2] . However, we did not check for the presence of skin inflammations and the yeast cells were seen in less than $15 \%$ of the patients. The impact of intestinal microbiota on Th17 in the CNS investigated by other researchers [23] were not also looked at.

From our questionnaires, we determined that epileptic seizures have seriously impaired a good number $(46.3 \%$ or $38 / 82)$ of the patients in Ngie. Some have suffered serious burns wherein they have lost parts of their bodies (e.g. hand, leg or fingers amputated). Others have suffered injuries from hard/sharp objects during attacks and $95.1 \%$ of them are suffering from some sort of mental retardation. Place of birth (at home, health Centre, hospital or dispensary) did not influence or determine whether an individual will be epileptic or not.

This lack of association of epilepsy with several environmental risk factors which we looked at in this study only shows that epilepsy in Ngie may be linked to causes other than those investigated in this study.

\section{Conclusion}

A high prevalence of epilepsy was observed in a selection of clusters in Ngie with varying cluster-specific levels of prevalence. However, these were not found to be associated with taeniasis. A positive family history and fungal infection may be associated with epilepsy in Ngie. Characteristics of onchocerciasis associated epilepsy were also observed in the results. Future studies on human genetic, fungal and onchocerciasis associated factors may unravel the actual cause of epilepsy in this area.

\section{Limitations}

Epilepsy status was only derived from questionnaires and some patients not comfortable with the stigma associated with the disease might have refused to declare their correct status. Our case control study population consisted only volunteers (could be biased). The tendency to only include patients with visible epilepsy (tonic-clonic seizures) and not those with less visible epilepsy (absences), no use of medical professionals to determine the kind of epilepsy (and whether it truly was epilepsy). We did not do assessment of Onchocerca volvulus infection.

\section{Acknowledgements}

We thank the administration and staff of Andek, Teze, Ajei, and Tinechung Integrated health centers for providing space and equipment in their laboratory units for this piece of work. We are grateful to Chief Dr. Azobi Vincent, the study participants and all who devoted precious time to discuss the epilepsy situation in Ngie with us and their advice on how to interact with the entire population during the study.

\section{Competing Interests}

The authors declare that they have no competing interest.

\section{Author Contributions}

Conceived and designed the experiment: Anong Damian Nota, Samuel Wanji, Raphael Awah Abong, Theresa Nkuo-Akenji, Peter Ndefon, Tatsinkou Betrand Fossi; Administration of questionnaires: Raphael Awah Abong, Ayong Hassan Fongoh; Sample collection and examination: Raphael Awah Abong, Ayong Hassan Fongoh, Anong Damian Nota; Analyzed the data: Raphael Awah Abong; Wrote the paper: Raphael Awah Abong, Anong Damian Nota, Samuel Wanji; Contributed to improving the manuscript: Theresa Nkuo-Akenji, Peter Ndefon, Tatsinkou Betrand Fossi.

\section{References}

1. Fisher RS, Van Emde Boas W, Blume W, Elger C, Genton P, et al. (2005) Epileptic seizures and epilepsy: Definitions proposed by the International League Against Epilepsy (ILAE) and the International Bureau for Epilepsy (IBE). Epilepsia 46: 470-472.

2. Vezzani A, Fujinami RS, White HS, Preux PM, Blumcke I, et al. (2016) Infections, inflammation and epilepsy. Acta Neuropathol 131: 211-234.

3. WHO (2018) Epilepsy. World Health Organization.

4. Ba-Diop A, Marin B, Druet-Cabanac M, Ngoungou EB, Newton CR, et al. (2014) Epidemiology, causes, and treatment of epilepsy in sub-Saharan Africa. Lancet Neurol 13: 1029-1044.

5. Zoli A, Shey-Njila O, Assana E, Nguekam JP, Dorny P, et al. (2003) Regional status, epidemiology and impact of Taenia solium cysticercosis in Western and Central Africa. Acta Trop 87: 35-42.

6. Del Brutto $\mathrm{OH}$, Santibanez R, Idrovo L, Rodriguez S, Diaz-Calderon E, et al. (2005) Epilepsy and neurocysticercosis in Atahualpa: A door-to-door survey in rural coastal Ecuador. Epilepsia 46: 583-587.

7. Gripper LB, Welburn SC (2017) The causal relationship between neurocysticercosis infection and the development of epilepsy - A systematic review. Infect Dis Poverty 6: 31.

8. Mukaratirwa $S$, Kassuku AA, Willingham AL, Murrell KD 3RD (2003) Background to the international action planning workshop on Taenia solium cysticercosis/taeniosis with special focus on Eastern and Southern Africa. Acta Trop 87: 3-5. 
9. Nguekam JP, Zoli AP, Zogo PO, Kamga AC, Speybroeck N et al. (2003) A seroepidemiological study of human cysticercosis in West Cameroon. Trop Med Int Health 8: 144-149.

10. Zoli AP, Nguekam, Shey-Njila O, Nsame Nforninwe D, Speybroeck N, et al. (2003) Neurocysticercosis and epilepsy in Cameroon. Trans R Soc Trop Med Hyg 97: 683-686.

11. Pion SD, Kaiser C, Boutros-Toni F, Cournil A, Taylor MM, et al. (2009) Epilepsy in onchocerciasis endemic areas: Systematic review and meta-analysis of population-based surveys. PLoS Negl Trop Dis 3: e461.

12. Colebunders R, Njamnshi AK, Van Oijen M, Mukendi D, Kashama JM, et al. (2017) Onchocerciasis-associated epilepsy: From recent epidemiological and clinical findings to policy implications. Epilepsia Open 2: 145-152.

13. Njamnshi AK, Sini V, Djientcheu VP, Ongolo ZP, Mapoure $Y$, et al. (2007) Risk factors associated with epilepsy in a rural area in Cameroon: A preliminary study. African Journal of Neurological Sciences 26: 18-26.

14. Prischich F, De Rinaldis M, Bruno F, Egeo G, Santori C, et al. (2008) High prevalence of epilepsy in a village in the Littoral Province of Cameroon. Epilepsy Res 82: 200-210.

15. Wanji S, Tendongfor N, Esum M, Yundze SS, Taylor MJ, et al. (2005) Combined utilisation of rapid assessment procedures for Loiasis (RAPLOA) and Onchocerciasis (REA) in rain forest villages of Cameroon. Filaria J 4: 2.
16. Andek council (2014) About Andek Council. Andek Council, Momo Division-Cameroon.

17. Azobi (1979) Azobi Vincent. Map and Geographical location of Ngie clan. Archives: Ngie council library.

18. Hoshaw-Woodard S (2001) Description and comparison of the methods of cluster sampling and lot quality assurance sampling to assess immunization coverage. WHO, Geneva.

19. Cheesbrough M (2006) District Laboratory Practice in Tropical Countries. Part 2, Cambridge University Press.

20. (1994) Relation between epilepsy and tropical diseases. Commission on tropical diseases of the International League Against Epilepsy. Epilepsia 35: 89-93.

21. Colebunders R, Mandro M, Mokili JL, Mucinya G, Mambandu G, et al. (2016) Risk factors for epilepsy in Bas-Uele Province, Democratic republic of the Congo: A case-control study. Int J Infect Dis 49: 1-8.

22. Kaiser C, Asaba G, Kasoro S, Rubaale T, Kabagambe G, et al. (2007) Mortality from epilepsy in an onchocerciasis-endemic area in West Uganda. Trans R Soc Trop Med Hyg 101: 48-55.

23. Wu J, Zhang $Y$, Yang $H$, Rao $Y$, Miao J, et al. (2016) Intestinal microbiota as an alternative therapeutic target for epilepsy. Can J Infect Dis Med Microbiol 2016: 9032809. 